\title{
PENSATA
}

DOI: http://dx.doi.org/10.1590/So034-759020140209

\section{ESTETIZAÇÃO DO MARKETING}

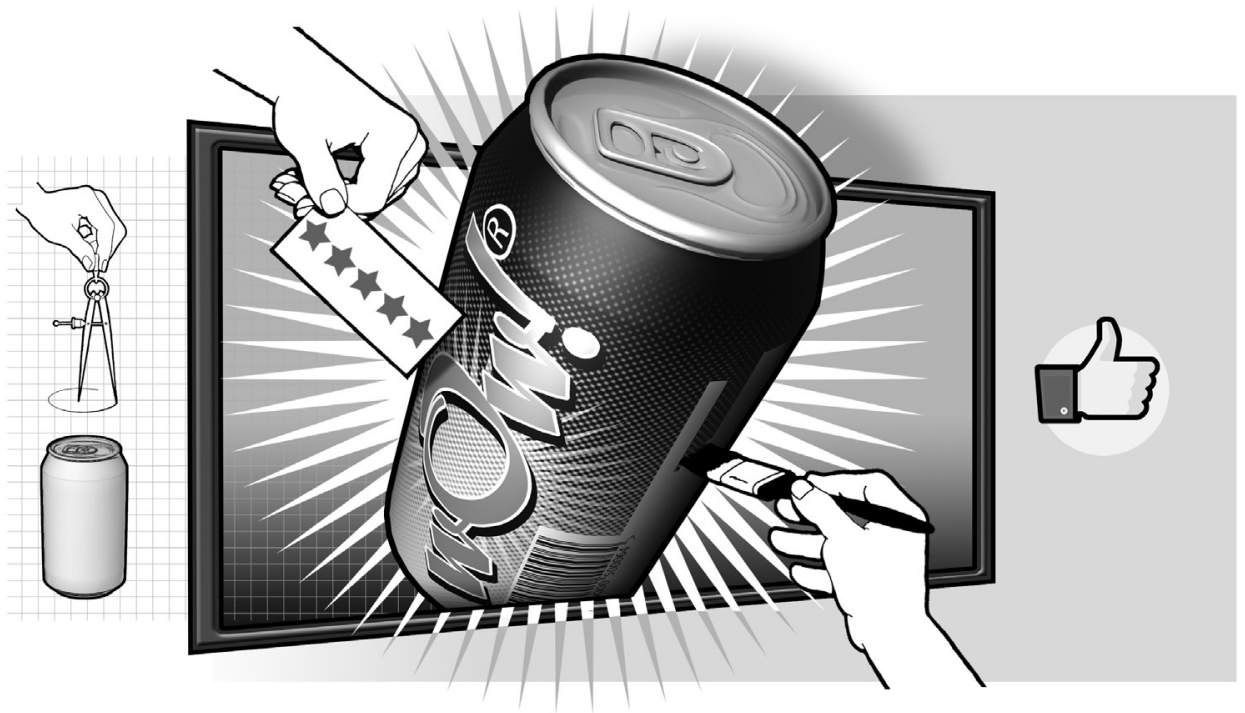

\section{ESTETIZAÇÃO DA SOCIEDADE}

\section{RICARDO TEIXEIRA VEIGA}

ricardo.necc@gmail.com

Professor da Faculdade de Ciências

Econômicas, Universidade Federal

de Minas Gerais, Belo Horizonte,

$M G$ - Brasil

\section{ANDRÉ TORRES URDAN}

andre.urdan@gmail.com

Professor da Escola de Administração de Empresas de São Paulo, Fundação Getulio Vargas, São Paulo, SP - Brasil

\section{CELSO AUGUSTO DE MATOS}

celsoam@unisinos.br

Professor do Centro de Ciências

Econômicas, Universidade do Vale do

Rio dos Sinos, São Leopoldo, RS - Brasil
A distopia imaginada por George Orwell no romance 1984 descreve o pesadelo de viver numa ditadura tecnologicamente aparelhada. 0 monitoramento do cotidiano é feito por câmeras de televisão. Praticamente não há lugar livre de vigilância constante, o que transforma atos banais, como escrever um diário, em comportamentos suspeitos. 0 que não é visto eletronicamente é controlado pelos cidadãos, adestrados para identificar subversivos e entregá-los à polícia. No livro, a tecnologia também é usada para mudar a percepção que os cidadãos têm da realidade, mantendo-os convencidos de que o presente é melhor do que o passado, apesar da saúde precária, frio e contínuas quedas nas rações de comida.

Em 1984, é especialmente interessante o tipo de trabalho feito no "Ministério da Verdade". Winston, protagonista do livro, refaz fotografias e reescreve trechos de notícias publicadas, para que os registros oficiais do passado não contradigam as posições correntes do governo. Como extensão sinistra dessa modelagem, nas referências ao dicionário da Novilíngua, Orwell especula sobre as possibilidades de controle do próprio pensamento, pela supressão de palavras e conceitos, simplificação da sintaxe e criação de neologismos, ideologicamente orientados.

0 retoque de fotografias faz-nos pensar no Photoshop ${ }^{\text {TM }}$. Sabemos que as imagens perfeitas das revistas não são reais, pois superam "defeitos" da realidade. São imagens rearranjadas e transformadas, por meio da manipulação do conteúdo binário das fotos.

Esse potencial de moldar a matéria conforme intenções estéticas, não como processo de imitação, mas como função produtiva, é que o filósofo Wolfgang Welsch chama de "estetização profundamente assentada” (Welsch, 1996). Trata-se de um movimento que atua nos fundamentos da sociedade. Está presente na prototipagem de produtos e na configuração de linhas de produção por meio de comandos na tela do computador. É a estetização evidente no uso de impressoras $3 \mathrm{D}$, capazes de produzir artefatos funcionais com base na impressão 
de imagens digitais. 0 autor comenta que o monitor da indústria mostra a capacidade de moldar-se a matéria conforme intenções estéticas, não como processo de imitação, mas como função produtiva. Esse movimento evidencia a tendência geral de priorização da estética. Resulta do uso da microeletrônica, estende-se a outros domínios, com a biotecnologia, e atingirá resultados, com a nanotecnologia, hoje considerados inacreditáveis. Essa estetização material acarreta outra, de natureza imaterial, caracterizada pela consciência, adquirida por quem constantemente trabalha com projetos apoiados por computadores, de como a realidade é virtual e esteticamente modelável.

Welsch (1996) denomina como "estetização superficial” um segundo amplo movimento, mais facilmente visível, porém menos importante teoricamente, caracterizado pela busca da sociedade por embelezamento, estilização e promoção de experiências.

Na sociedade de consumo, a estetização superficial é conspícua nos cuidados esmerados da arquitetura e decoração, nos investimentos elevados no design de produtos, marcas e websites. É a estetização evidente nas emocionantes experiências coletivas, no cinema 3D e nas recentes TVs de altíssima definição. Preocupação exagerada com aparência e estilização manifesta-se frequentemente na maneira como as pessoas se vestem e cuidam de seu próprio corpo, em busca da aparência física perfeita.

A estetização das experiências é também descrita no livro de Orwell. Filmes artisticamente trabalhados, com enredos simplórios, mas de forte apelo emocional, levam multidões ao ufanismo delirante, ódio e xenofobia, motivando-as permanentemente para a guerra. Em importância e natureza, esses filmes aludem à comoção coletiva dos grandes comícios políticos e à mobilização induzida pela qualidade primorosa dos filmes de Leni Riefenstahl (1902-2003), realizados para promover o nazismo. Em magnitude de apelo emocional e engajamento, lembram grandes celebrações religiosas e shows de música pop do século XX.

Os dois movimentos gerais de estetização identificados por Welsch (1996) generalizam os processos sugeridos no livro 1984 e revelam a tendência geral de priorização da estética na sociedade de consumo contemporânea. Demonstram a potência produtiva e revolucionária das novas tecnologias de informação. Indicam novos meios de percepção e modificação da realidade. Repercutem em nossas crenças, valores, hábitos e estilos de vida.

Mudamos de canal, marcas ou produtos quando estamos insatisfeitos ou entediados. A indústria cultural capitalista domina a cultura de massa, seduzindo-nos pelas novidades, vendendo-nos continuamente a promessa do espetáculo (Adorno \& Horkheimer, 1984; Debord, 1983), tanto no lazer quanto na informação. Para motivar ou prender nossa atenção, tudo é apresentado como extraordinário ou sensacional. 0 que experimentamos, porém, mesmo nas notícias, corresponde ao produto de edições cuidadosas de fatos e imagens, que se sucedem e se multiplicam vertiginosamente. A sociedade do espetáculo permanente confunde-se com a sociedade da informação. Durante grande parte do tempo, estamos imersos na realidade virtual da internet, programas de computadores, videogames e redes sociais.

Para Baudrillard (1991), esse ambiente cultural estetizado das sociedades de consumo é uma "hiper-realidade". Nela, tanto desaparecem as distinções entre objeto e imagem, entre fato e representação, quanto se sobrevalorizam imagens e representações relativamente a seus correspondentes reais. Segundo o autor, o ambiente tornou-se uma realidade alucinatória, fundada na experiência sensorial hipertrofiada e de imersão, característica da mudança radical de nossa forma de perceber o mundo e com ele nos relacionarmos, induzida pelos meios de comunicação de massa e pela tecnologia.
Os dois tipos de estetização apontados por Welsch refletem, também, a permeabilização das fronteiras entre a arte e outras formas culturais de expressão. De modo mais específico, a estetização da sociedade totalitária do romance de Orwell pode ser interpretada como “estetização da política”. Esse processo foi originalmente definido por Benjamin (2000) como uso da arte, especialmente fotografia e cinema, para promover ideologias e governos fascistas. 0 conceito foi posteriormente estendido para descrever a promoção de qualquer tipo de ideologia política por meio da arte ou técnicas artísticas, como se faz hoje vulgarmente nas democracias modernas, utilizando-se televisão e internet, como no caso da vitoriosa campanha de Barack Obama, para presidente dos Estados Unidos, em 2008.

A instigante visão de Welsch sobre estetização pode ser comparada à de Mike Featherstone (1991), que aborda os múltiplos sentidos da estetização da vida cotidiana. Primeiramente, Featherstone refere-se à reação do Surrealismo e outros movimentos artísticos europeus do início do século XX à institucionalização do modernismo em museus e academias. Esses movimentos buscavam dessacralizar a arte tradicional, retirando seu halo de respeitabilidade, associado a seu cultivo em museus, galerias e outros espaços restritos. Objetos comuns foram promovidos a peças de arte, por meio da aplicação do conceito de ready-made, criado pelo francês Marcel Duchamp (1887-1968), que gerou perplexidade e revolta ao assinar um urinol, comprado em loja especializada, como obra de arte, expondo-o numa galeria dos Estados Unidos. Em coerência com esse ataque à arte convencional, popularizaram-se as performances - apresentações transitórias que não podem ser transformadas em peças de museu.

O colapso de fronteiras e distinções entre arte e vida é constatado em pesquisas sobre arte pop e estudos sobre aplicação de técnicas e padrões artísticos em campanhas 
publicitárias e criação de valor de marca (Szmigin, 2006). A autora discute implicações da convergência entre arte e consumo, para produtores e consumidores. Estes não apenas são encorajados a integrar o consumo e a experiência estética, aceitando a fluidez e ruptura da distância entre as duas esferas, mas também passam a dominar conceitos e um vocabulário básico que lhes permitem transitar entre apreciação estética e vivência instrumental. Por outro lado, produtores aumentam seu potencial de manipulação imagética e de signos, à medida que utilizam técnicas e recursos baseados na arte elevada e no design gráfico, em enfoques inovadores aceitáveis pelo mercado, promovendo experiências integrativas de consumo, por meio da comunicação, gestão da ambiência das lojas e design dos produtos.

Como segundo tipo da estetização do cotidiano, Featherstone aponta o projeto de transformar a vida pessoal em obra de arte, ética de vida promovida por artistas como Oscar Wilde e endossada por filósofos como Foucault e Rorty. 0 estilo de vida buscado podia ser notado no dandismo e devia centrar-se na fruição estética e expansão da mente por meio da experimentação constante de novos gostos e sensações.

O terceiro e último significado da estetização do cotidiano apontado por Featherstone refere-se ao predomínio do rápido fluxo de signos e imagens no tecido da vida habitual na sociedade contemporânea. Apoiando-se no conceito marxista de fetichismo da mercadoria, Adorno concluiu que a dominação do abstrato valor de troca sobre o concreto valor funcional liberou as mercadorias para assumirem um valor de uso secundário, denominado, por Baudrillard, como sígnico (Featherstone, 1991).

$\mathrm{Na}$ sociedade de consumo, segundo Baudrillard (2008), a partir de um patamar mínimo de satisfação das necessidades elementares, o valor de uso dos produtos perde sua importância como principal justificativa de consumo. É superado pelo valor de troca ou de mercado. Em seu sistema de va- lor, Baudrillard associa a produtos também valor simbólico e valor de signo. Valor simbólico corresponde ao valor atribuído a um objeto em função de seu significado subjetivo ou representação social. Como presente recebido, por exemplo, um produto pode tornar-se insubstituível; uma aliança de casamento simboliza fidelidade. Valor sígnico é o valor de um objeto em relação a outros, que compõem um sistema ou constelação, tal como a referência de maior prestígio conferido à posse do mais novo iPhone ${ }^{\mathrm{TM}}$ da Apple, comparado a modelos anteriores da mesma marca ou a smartphones de outros fabricantes. Como signos, objetos de uma categoria são substituíveis por objetos de outras, por exemplo, uma distinção acadêmica pode ser equivalente ou superar um diploma como signo de competência ou notório saber. Numa diferença relativa à sociedade tradicional, Baudrillard ressalta que, na sociedade de consumo, mercadorias são trocadas como signos, porque supostamente os objetos sempre comunicam algo sobre seus usuários.

Com a produção ilimitada de bens, sucessão de ciclos de moda cada vez mais curtos, obsolescência planejada e desejo obcecado dos consumidores por lançamentos e inovações, a referência de valor sígnico muda constantemente, desafiando as bases correntes de luxo, poder e distinção. Em função da rápida obsolescência do design, muitos produtos são percebidos como ultrapassados mesmo antes de perderem seu valor funcional.

Devido aos amplos e óbvios impactos da estetização na cultura e estrutura social, com repercussão direta nos fenômenos de troca, é necessário entender como a base de conhecimentos de marketing tem sido atualizada para adequar-se à realidade contemporânea.

\section{MARKETING E ESTETIZAÇÃO: INFLUÊNCIA RECÍPROCA}

A aplicação de conhecimentos e técnicas de marketing potencializa os efeitos da es- tetização da política. Pesquisa de mercado, segmentação, posicionamento, estratégia e plano de marketing são processos e conceitos-chave para eleger candidatos e gerenciar a imagem de políticos e partidos. Os resultados combinados do marketing com estetização são frequentemente campanhas publicitárias artisticamente sofisticadas e muito eficazes.

Essa combinação bem-sucedida é utilizada em vários domínios. Conhecimentos de marketing embasam o efetivo uso das mídias eletrônicas no desenvolvimento e promoção de marcas e produtos. A estetização da sociedade aprofunda-se pelo impulso da orientação de mercado e práticas de marketing alinhadas com valores hedônicos e imediatistas, contribuindo para reduzir o cidadão à condição de cliente, enfraquecendo a cidadania e estimulando o consumismo (Bauman, 2008; Hutton, 2005), mas também levando os governos a preocuparem-se com a melhora da oferta de serviços públicos, mirando-se na excelência de serviços privados (Mosse \& Whitley, 2009).

0 que é artisticamente trabalhado cativa atenção, revitaliza a experiência, aumenta a percepção de valor e reforça o consumo. Resultados econômicos das indústrias criativas são bastante influenciados pelas mudanças de natureza estética (soft innovation) dos produtos (Stoneman, 2010) e orçamento de marketing disponível para sua promoção e distribuição (Hennig-Thurau, Houston \& Sridhar, 2006; Ravid, 1999). $\mathrm{Na}$ indústria eletrônica de bens de consumo e automobilística, por exemplo, o design é fator destacado na decisão de compra e satisfação dos consumidores, especialmente das mulheres (Beck, 2011).

Avanços metodológicos e teóricos de marketing acompanham a estetização da sociedade e resultam da aplicação das novas tecnologias da informação às atividades de marketing (Strauss \& Frost, 2012). Marketing digital ou $e$-marketing pode ser conceituado como a integração de recursos 
complementares de tecnologia, pessoal e negócios, cuja combinação, quando centrada na orientação de mercado e de tecnologia, influencia positivamente o desempenho das empresas, por meio da retenção e satisfação de clientes (Trainor, Rapp, Beitelspacher \& Schillewaert, 2011). Ações de vendas, propaganda, marketing direto, relações públicas, prestação de serviços, pesquisa de mercado e relacionamento são feitas pela internet, utilizando-se as possibilidades de comunicação e substituição de processos e produtos físicos por sucedâneos virtuais.

O neuromarketing - aplicação de métodos de imagens neurais no marketing de produtos - vem ganhando considerável popularidade, por causa da tendência de ficar mais barato e tornar-se mais eficiente, e principalmente devido ao seu potencial de revelar informações ocultas sobre a experiência do consumidor, inacessíveis a métodos convencionais (Ariely \& Berns, 2010).

0 escaneamento cerebral pode mostrar os fundamentos de estratégias de marketing bem e mal sucedidas, novas oportunidades de posicionamento e chaves para a garantia de sucesso da persuasão na publicidade. Com a demonstração consistente de nexos causais entre reações cerebrais específicas e respostas emocionais e cognitivas de consumidores às estratégias de marketing, neuroimagens poderão ser sistematicamente usadas em sistemas de prototipagem e simulação, contribuindo para a produção de ofertas de mercado bem-sucedidas. Relativamente ao seu potencial produtivo, neuromarketing é, assim, exemplo da estetização profunda da sociedade, na perspectiva de Welsch (1996).

Apesar da influência benéfica do marketing na promoção de uma cultura afluente, democrática e liberal (Baker, 2010), nas sociedades capitalistas estetizadas, o materialismo (Kasser, 2002), consumo exagerado e preocupação ecológica insuficiente tendem a prevalecer (Taylor \& Tilford, 2000). Essa situação é acentuada pelo uso profissional e efetivo de conceitos e métodos de marketing para promover interesses econômicos e políticos, reforçando os valores convencionais capitalistas e da sociedade de consumo (Baudrillard, 2008; Bauman, 2008; Friedan, 1963).

Há também movimentos sociais de resistência à estrutura de dominação subjacente à pressão para consumir, denominados resistência do consumidor e anticonsumo (Cherrier, Black \& Lee, 2011; lyer \& Muncy, 2009; Kozinets, Handelman \& Lee, 2010; Pentina \& Amos, 2011), cuja incorporação de princípios à base teórica de marketing tem sido debatida (ver, por exemplo, Lee, Roux, Cherrier \& Cova, 2011). Autores consagrados têm buscado atualizar a disciplina à realidade dos novos tempos. Por exemplo, há muitos anos, Kotler (1982) advoga o conceito de marketing societal, que destaca a necessidade de atendimento de amplos interesses sociais, como qualidade de vida e sustentabilidade, ultrapassando demandas imediatas das partes diretamente envolvidas nas trocas. Andreasen (1994) propôs revisão do conceito de marketing social, interpretando-o como uso de conceitos e métodos de marketing para influenciar o comportamento voluntário das pessoas, promovendo seu bem-estar e da coletividade. Na prática, entretanto, é difícil conciliar ganhos individuais e sociais, porque o sistema de marketing está inserido nas rotinas e expectativas da sociedade, tanto refletindo seus pressupostos e prioridades quanto influenciando como indivíduos e organizações percebem as questões sociais contemporâneas e atuam sobre elas (Matear \& Dacin, 2010).

\section{Lógica Dominada pelo Serviço como Reflexo da Estetização da Sociedade}

Como ciência comportamental, o marketing estuda os processos gerais de troca, considerando perspectivas socioeconômicas, psicológicas e culturais (Hunt, 2002). Recente avanço teórico da disciplina indica uma mu- dança de orientação: a passagem de uma lógica baseada em produto para uma lógica dominada pelo serviço (LDS), com possibilidades de constituir-se na base de uma teoria geral da área (Vargo \& Lusch, 2004). A LDS simplifica o modelo de troca, unificando o pensamento de marketing do consumidor e industrial, e redefinindo a utilidade das trocas em termos de benefícios recebidos, subordinando o valor de troca ao valor emergente no uso (Vargo \& Lusch, 2008).

$\mathrm{Na}$ lógica predominante anterior, produto era o conceito básico, caracterizado como objeto destinado a satisfazer necessidades ou desejos de consumidores ou usuários (Kotler, 1999). Nessa acepção, consideravam-se produtos tanto bens físicos quanto serviços, entendidos esses como atividades ou processos, ou seja, bens imateriais. Para fins gerenciais, enfatizava-se a distinção de serviços em relação a bens, considerando-se aspectos como grau de tangibilidade, potencial de separar a produção da distribuição, possibilidade de garantir qualidade homogênea dos itens produzidos e de estocar-se, para venda posterior, a produção ofertada não consumida (Zeithaml, 1981). Na prática, porém, são comuns ofertas de marketing em que se associam bens e serviços, bem como outras entidades cuja classificação nesses dois tipos básicos é controversa (por exemplo, software, informação e conhecimento). Por isso, desenvolvimento teórico recente no marketing passou a enfatizar a convergência de bens e serviços, em vez dos aspectos que os diferenciam intrinsecamente.

A LDS centra-se na ideia unificadora de serviço (termo utilizado no singular). Serviço é a aplicação de competências especializadas (isto é, conhecimentos e habilidades), por meio de ações, processos e performances, visando ao benefício da outra parte (Vargo \& Lusch, 2008). Na nova orientação, bens, serviços específicos ou suas associações são abordados como suporte para a entrega de benefícios. Por conseguinte, não importa qual seja seu nível 
de desenvolvimento e sofisticação, todas as economias e negócios devem ser considerados economias e negócios de serviço.

Pensar no marketing dominado pela ideia de serviço é inerentemente pensar o marketing como orientado aos consumidores e relacional, o que reforça a passagem da mentalidade de marketing transacional para o de relacionamento. Uma premissa fundamental da LDS é que valor é produzido na cooperação entre produtos e consumidores, por meio da integração de recursos. Por isso, uma empresa não pode garantir a entrega de valor, mas apenas oferecer proposições de valor, o qual é singular e fenomenologicamente determinado pelos beneficiários. Por exemplo, se fabricantes utilizam embalagens recicláveis em seus produtos industrializados, é necessário que as autoridades governamentais ofereçam programas de reciclagem que engajem os consumidores, para que a proposta de valor se concretize.

O conceito unificante de serviço resulta do influxo da tecnologia da informação e da sociedade de consumo, concretizado nos processos de estetização. Devido à transformação de conteúdo físico em conteúdo informacional, a flexibilidade, integração e portabilidade dos produtos aumentam cada vez mais, como resultante da nova infraestrutura provida pela tecnologia de informação para as atividades produtivas e comunicativas, em seu potencial de automatizar e informatizar (Zuboff, 1994). “Serviço", assim, corresponde à possibilidade de síntese de atividades de produção, gestão e consumo, com base em sistemas inteligentes e tecnologias de informática e comunicação cada vez mais potentes e amplamente disponíveis.

A LDS baseia-se no afrouxamento da distinção entre produtores e consumidores, bem como dos limites entre produção e consumo, e pode ser tomada como típica da transição para uma sociedade pósmoderna e pós-industrial. De fato, a cunhagem, em 1980, por Alvin Toffler, do termo prosumer, junção de produtor e consumidor (Toffler, 2001), indicou a emergência de consumidores menos conformistas, cujo comportamento e comunicação, na internet e em outros meios interativos, afetam ativamente a evolução de produtos e ofertas de mercado, por meio da emissão de feedback, esclarecimento e mobilização de outros consumidores, produção espontânea de serviços, conteúdo informativo e cooperação voluntária nos esforços empresariais de pesquisa e desenvolvimento. As interações, em blogs jornalísticos, com os articulistas e apoio no desenvolvimento de verbetes da Wikipedia ilustram a atuação de "prossumidores".

O nivelamento entre produtores e consumidores é também criticado. Keen (2007) denuncia a proliferação de pseudoespecialistas na internet e a patente desvalorização do conhecimento profissional, por exemplo, na perda de prestígio de publicações especializadas e tradicionais para publicações colaborativas, como ocorreu com a popularização da Wikipedia, em detrimento da autoridade de enciclopédias como a Britânica. Scruton (2009) critica o abandono da beleza como valor artístico essencial, em detrimento da priorização do "gesto artístico", calcado no improviso e estetização do que é feio, extravagante ou banal. O filósofo aponta a perda da diferença qualitativa entre artista e espectador como uma das causas dessa decadência.

Por outro lado, a presunção de que, nas sociedades modernas, consumidores tendem a tornar-se mais informados, conscientes e autônomos, com poder de resistir ao assédio do marketing das empresas, definindo o que vão comprar ou adotando comportamentos anticonsumistas, contradiz pressupostos de teorias críticas do capitalismo.

A esse respeito, é exemplar a obra de autores da Escola de Frankfurt. Adorno e Horkheimer (1984) criticam o processo de massificação cultural, promovido por agências do capitalismo monopolista, organizadas em moldes industriais semelhantes aos de ramos tradicionais da economia, constituindo a indústria cultural de filmes, músicas, jogos, telenovelas, livros e produtos correlatos. Conforme essa teoria, consumidores são subjugados, seduzidos e manipulados, aderindo irrefletidamente a padrões de consumo e de gosto estabelecidos pela indústria cultural, num processo de estetização promovido à sua revelia. Por isso, a grande maioria do público prefere a produção da cultura de massa, descartando ou ignorando obras artisticamente mais sofisticadas ou com conteúdo mais crítico ou esteticamente inovador.

Debord (1983) critica dialeticamente o modo capitalista de organização social, que provoca alienação, apresentando possíveis estratégias de resistência aos valores e ideologia burguesa. Segundo o autor, como constituição moderna da luta de classes, o "espetáculo" - representação virtual e estetizada do mundo - reflete a dominação da burguesia sobre o proletariado e o resto da sociedade, promovendo a unificação cultural. Compreendido em sua totalidade, segundo o autor, o espetáculo é, ao mesmo tempo, resultado e projeto do modo de produção existente.

Banalizada nos meios de comunicação, a espetacularização da realidade, analisada por Debord, pode ser entendida como forma de estetização. Tudo que anteriormente era apenas concreta e diretamente vivido passa a ser vivenciado à distância, como representação. Por exemplo, notícias de perdas familiares são exploradas como dramas nos jornais televisivos: choros são reproduzidos com esmero artístico por meio de imagens em close, fartamente repetidas em diferentes canais e horários. A preferência pelo espetáculo também é evidente nas mudanças de hábitos de torcedores esportivos. Atualmente, grande parte do público prefere assistir a esportes televisionados a ir às arenas. A experiência visual mediada pela televisão é supostamente muito mais rica, devido à riqueza de detalhes provida por dezenas de câmaras, estatísticas, re- 
petição de cenas, câmera lenta, tira-teima etc., ou seja, a "representação é muito melhor do que a realidade", como atestam cartões-postais e fotos retocadas. Nas sociedades de consumo pós-modernas, esse ambiente cultural estetizado é a hiper-realidade anunciada por Baudrillard, cujos fenômenos de troca devem ser abarcados pela teoria de marketing.

Como paradigma emergente, a LDS intrinsecamente reconhece e incorpora princípios consistentes com os processos de estetização, entendidos, na perspectiva de Welsch (1996), como duplo movimento: de natureza mais superficial e de natureza profundamente assentada. Parece faltar à nova lógica, entretanto, uma perspectiva mais afinada com insights de teorias críticas da sociedade do consumo e do valor, que oferecem arcabouço alternativo para se entenderem os fenômenos de marketing na sociedade atual.

\section{TENDÊNCIAS EVOLUTIVAS DO MARKETING}

As leis de Mendel, elaboradas com base em experimentos com a reprodução de diferentes espécies de ervilhas, demarcaram os primórdios de um processo radical de mudança da biologia, transformada, pela genética, em tecnologia da informação (Dawkins, 2008). Baudrillard (2008) sugeriu inovação similar, avaliando a sociedade de consumo no ponto de vista de uma teoria da cultura e da comunicação.

Inovações metodológicas e teóricas do marketing parecem indicar sua transformação acelerada em ciência dura, refletindo movimentos gerais de estetização da sociedade. 0 desenvolvimento do marketing digital e do neuromarketing reforçam a natureza aplicada e experimental da disciplina. O uso intensivo da tecnologia, associado à base teórica interdisciplinar, aproxima o marketing da neurociência e da psicologia experimental, num processo de encontro com as ciências naturais. Para que conhe- cimentos obtidos no neuromarketing sejam incorporados à teoria de marketing, no entanto, o principal desafio é expandir e validar os resultados empíricos obtidos, bem como utilizar um enfoque crescentemente dedutivo, que permita revisar as teorias econômicas existentes (Hubert, 2010; Vecchiato et al, 2010).

No marketing digital, é possível medir com maior precisão a efetividade das suas ações e programas. Nivelamento entre produtores e consumidores, convergência de mídias, sistemas e produtos, aumento da portabilidade e conectividade potencializam o alcance e resultados das estratégias de marketing.

A proposta de mudança de paradigma na teoria de marketing para a lógica dominada pelo serviço reflete as profundas mudanças tecnológicas, econômicas e culturais na sociedade da informação e do espetáculo. A redefinição de serviço na nova orientação é consequência natural do sombreamento das fronteiras entre realidade física e virtual e da sobrevalorização da dimensão estética no mundo contemporâneo.

Fundamental na teoria de marketing, a noção de valor como justificativa das trocas é reavaliada na LDS, concluindo-se que as ofertas de mercado constituem-se em "propostas de valor". Valor é um conceito complexo e polissêmico, consequentemente é necessário superar o pensamento simplista da economia tradicional de considerá-lo em termos da dicotomia entre valor de uso e de troca. Grönroos (2011) analisa a lógica subjacente ao princípio de cocriação de valor, sugerindo reformulações e extensões das premissas da LDS, com base na primazia da criação recíproca de valor nos negócios, atuando o serviço como mediador. 0 autor critica o dogmatismo na aceitação das proposições fundacionais da LDS sem o exame rigoroso de suas implicações teóricas e gerenciais.

A necessidade de assimilação de abordagens críticas dos fenômenos de troca e consumo indica a oportunidade do recurso a teorias da sociedade de consumo como a de Baudrillard (2008), aumento do uso de métodos qualitativos e aprofundamento dos esforços de teorização em marketing. A perspectiva do autor de pensar o valor também em termos simbólicos e de signos, bem como de considerar os fundamentos míticos da sociedade de consumo, é adequada ao marketing no contexto da sociedade de consumo estetizada e hiper-real.

A estetização geral da sociedade manifesta-se tanto nos métodos quanto na teoria de marketing. Consequentemente, é essencial reforçar sua interdisciplinaridade e vínculos com a filosofia e ciências da cultura, como a sociologia e a antropologia, para que as trocas sejam entendidas especialmente como fenômeno estético e cultural, cujas referências de valor mudam continuamente.

\section{REFERÊNCIAS}

Adorno, T, \& Horkheimer, M. (1984). Dialética do esclarecimento. (G. Almeida, Trad.). Rio de Janeiro: Zahar. (Obra original publicada em 1947).

Andreasen, A. R. (1994). Social marketing: its definition and domain. Journal of Public Policy \& Marketing, 13(1), 108-114.

Ariely, D, \& Berns, G. S. (2010). Neuromarketing: the hope and hype of neuroimaging in business. Nature Reviews Neuroscience, 11(4), 284-292.

Baker, M. J. (2010). Marketing: philosophy or function? In M. J. Baker \& M. Saren (Orgs.). Marketing theory: a student text (2nd ed, pp. 3-25). London: Sage.

Baudrillard, J. (2008). A sociedade de consumo. Lisboa: Edições 70. (Obra original publicada em 1970).

Baudrillard, J. (1991). Simulacro e simulações. Lisboa: Relógio D’Água. (Obra original publicada em 1981).

Bauman, Z. (2008). Vida para consumo: a transformação das pessoas em mercadoria. Rio de Janeiro: Zahar.

Beck, K. (2011). The feminine marketing mystique: how to demystify women's purchasing behavior. Consultado de http://www.nxtbook. com/nxtbooks/crmmedia/crmo411/index. php\#/18. 
Benjamin, W. (2000). A obra de arte na época de sua reprodutibilidade técnica. In T. Adorno (Org.). Teoria da cultura de massa. (pp. 221254). (C. N. Coutinho, Trad.). São Paulo: Paz e Terra. (Obra original publicada em 1936).

Cherrier, H, Black, I. R, \& Lee, M. (2011). Intentional non-consumption for sustainability: consumer resistance and/or anti-consumption? European Journal of Marketing, 45(11-12), 1757-1767.

Dawkins, R. (2008). The Oxford book of modern science writing. Oxford: Oxford University Press.

Debord, G. (1983). Society of the spectacle. Detroit: Black and Red. (Primeira parte da obra publicada em 1967).

Featherstone, M. (1991). Consumer culture and postmodernism (2nd ed.). London: Sage.

Friedan, B. (1963). The feminine mystique. New York: W. W. Norton \& Co.

Grönroos, C. (2011). Value co-creation in service logic: a critical analysis. Marketing Theory, 11(3), 279-301.

Hennig-Thurau, T, Houston, M. B, \& Sridhar, S. (2006). Can good marketing carry a bad product? Evidence from the motion picture industry. Marketing Letters, 17(3), 205-219.

Hubert, M. (2010). Does neuroeconomics give new impetus to economic and consumer research? Journal of Economic Psychology, 31(5), 812-817.

Hunt, S. (2002). Foundations of marketing theory: toward a general theory of marketing. New York: M. E. Sharpe.

Hutton, J. G. (2005). The feel-good society: how the "customer" metaphor is undermining American education, religion, media and healthcare. West Paterson: Pentagram Publishing.

Iyer, R, \& Muncy, J. A. (2009). Purpose and object of anti-consumption. Journal of Business Research, 62(2), 160-168.
Kasser, T. (2002). The high price of materialism. Cambridge: MIT Press.

Keen, A. (2007). The cult of the amateur: how blogs, MySpace, YouTube, and the rest of today's user-generated media are destroying our economy, our culture, and our values. Doubleday: New York.

Kotler, P. (1982). Marketing for non-profit organizations (2nd ed.). Upper Saddle River: Prentice Hall.

Kotler, P. (1999). Marketing para o século XXI: como criar, conquistar e dominar mercados. São Paulo: Futura.

Kozinets, R. V, Handelman, J. M, \& Lee, M. S. W. (2010). Don't read this; or, who cares what the hell anti-consumption is, anyway? Consumption Markets \& Culture, 13(3), 225-233.

Lee, M. S. W, Roux, D, Cherrier, H, \& Cova, B. (2011). Anti-consumption and consumer resistance: concepts, concerns, conflicts, and convergence. European Journal of Marketing, 45(11-12), 1680-1687.

Matear, M, \& Dacin, P. A. (2010). Marketing and societal welfare: a multiple stakeholder approach. Journal of business research, 63(11), 1173-1178.

Mosse, B, \& Whitley, E. A. (2009). Critically classifying: UK e-government website benchmark ing and the recasting of the citizen as customer. Information Systems Journal, 19(2), 149-173.

Pentina, I, \& Amos, C. (2011). The Freegan phenomenon: anti-consumption or consumer resistance? European Journal of Marketing 45(11-12), 1768-1778.

Ravid, A. (1999). Information, blockbusters, and stars: a study of the film industry. The Journal of Business, 72(4), 463-492.

Scruton, R. (2009). Beauty. Oxford: Oxford University Press.

Stoneman, P. (2010). Soft innovation: economics, product aesthetics, and the creative indus tries. Oxford: Oxford University Press.
Strauss, J, \& Frost, R. (2012). E-marketing (6a ed.). São Paulo: Pearson Prentice Hall.

Szmigin, i. (2006). The aestheticization of consumption: an exploration of 'brand.new' and 'shopping'. Maketing Theory, 6(1), 107-118.

Taylor, B, \& Tilford, D. (2000). Why consumption matters. In J. B. Schor \& D. B. Holt (Orgs.) The consumer society reader (pp. 463-487). New York: New Press.

Toffler, A. (2001). A terceira onda (26a ed.). Rio de Janeiro: Record.

Trainor, K. J, Rapp, A, Beitelspacher, L. K, \& Schillewaert, n. (2011). Integrating information technology and marketing: an examination of the drivers and outcomes of e-marketing capability. Industrial Marketing Management, 40(1), $162-174$.

Vargo, S. L, \& Lusch, R. F. (2004). Evolving to a new dominant logic for marketing. Journal of Marketing, 68(1), 1-17.

Vargo, S. L, \& Lusch, R. F. (2008). Why "service"? Journal of the Academy of Marketing Science, 36(1), 25-38.

Vecchiato, G, Fallani, F. V, Astolfi, L, Toppi, J, Cincotti, F, Mattia, D, Salinari, S, \& Babiloni, F. (2010). The issue of multiple univariate comparisons in the context of neuroelectric brain mapping: an application in a neuromarketing experiment. Journal of Neuroscience Methods, 191(2), 283-289.

Welsch, W. (1996). Aestheticization processes: phenomena, distinctions and prospects. Theory, Culture and Society, 13(1), 1-24.

Zeithaml, V. A. (1981). How consumer evaluation processes differ between goods and services. In C. Lovelock. Services marketing (2nd ed, p. 25-32). Upper Saddle River, New Jersey: Prentice Hall.

Zuboff, S. (1994). Automatizar / informatizar: as duas faces da tecnologia inteligente. RAE-Revista de Administração de Empresas, 34(6), 80-91. 